\title{
Smartphone App Using Mindfulness Meditation for Women With Chronic Pelvic Pain (MEMPHIS): Protocol for a Randomized Feasibility Trial
}

Elizabeth Ball ${ }^{1,2}, \mathrm{PhD}$; Sian Newton ${ }^{3}, \mathrm{MSc}$; Brennan C Kahan ${ }^{4}, \mathrm{MSc}$; Gordon Forbes ${ }^{4}, \mathrm{MSc}$; Neil Wright ${ }^{5}, \mathrm{PhD}$; Clara Cantalapiedra Calvete ${ }^{1}$, MPH; Harry A L Gibson ${ }^{1}$, MBBS; Ewelina Rogozinska ${ }^{2}$, MSc; Carol Rivas ${ }^{6}, \mathrm{PhD}$; Stephanie J C Taylor ${ }^{3}$, MD; Judy Birch ${ }^{7}$, BEd; Julie Dodds ${ }^{2}, \mathrm{PhD}$

\footnotetext{
${ }^{1}$ Department of Obstetrics and Gynaecology, Barts Health NHS Trust, London, United Kingdom

${ }^{2}$ Women's Health Research Unit, Barts and the London School of Medicine and Dentistry, Queen Mary University of London, London, United Kingdom

${ }^{3}$ Centre for Primary Care and Population Health, Queen Mary University of London, London, United Kingdom

${ }^{4}$ Pragmatic Clinical Trials Unit, Queen Mary University of London, London, United Kingdom

${ }^{5}$ Clinical Trial Service Unit and Epidemiological Studies Unit, University of Oxford, Oxford, United Kingdom

${ }^{6}$ Social Science Research Unit, University College London, London, United Kingdom

${ }^{7}$ Pelvic Pain Support Network, Poole, United Kingdom
}

Corresponding Author:

Elizabeth Ball, PhD

Department of Obstetrics and Gynaecology

Barts Health NHS Trust

Royal London Hospital

Whitechapel Road

London, E1 1BB

United Kingdom

Phone: 442073777000

Fax: 442073777000

Email: Elizabeth.ball@bartshealth.nhs.uk

\section{Abstract}

Background: Female chronic pelvic pain (CPP) is defined as intermittent or constant pelvic or lower abdominal pain occurring in a woman for at least 6 months. Up to a quarter of women are estimated to be affected by CPP worldwide and it is responsible for one fifth of specialist gynecological referrals in the United Kingdom. Psychological interventions are commonly utilized. As waiting times and funding capacity impede access to face-to-face consultations, supported self-management (SSM) has emerged as a viable alternative. Mindfulness meditation is a potentially valuable SSM tool, and in the era of mobile technology, this can be delivered to the individual user via a smartphone app.

Objective: To assess the feasibility of conducting a trial of a mindfulness meditation intervention delivered by a mobile phone app for patients with CPP. The main feasibility objectives were to assess patient recruitment and app adherence, to obtain information to be used in the sample size estimate of a future trial, and to receive feedback on usability of the app.

Methods: Mindfulness Meditation for Women With Chronic Pelvic Pain (MEMPHIS) is a three-arm feasibility trial, that took place in two hospitals in the United Kingdom. Eligible participants were randomized in a 1:1:1 ratio to one of three treatment arms: (1) the intervention arm, consisting of a guided, spoken mindfulness meditation app; (2) an active control arm, consisting of a progressive muscle relaxation app; and (3) usual care (no app). Participants were followed-up for 6 months. Key feasibility outcomes included the time taken to recruit all patients for the study, adherence, and estimates to be used in the sample size calculation for a subsequent full-scale trial. Upon completion of the feasibility trial we will conduct focus groups to explore app usability and reasons for noncompliance.

Results: Recruitment for MEMPHIS took place between May 2016 and September 2016. The study was closed March 2017 and the report was submitted to the NIHR on October 26, 2017.

Conclusions: This feasibility trial will inform the design of a large multicentered trial to assess the clinical effectiveness of mindfulness meditation delivered via a smartphone app for the treatment of CPP. 
Trial Registration: ClinicalTrials.gov: NCT02721108; https://clinicaltrials.gov/ct2/show/NCT02721108 (Archived by WebCite at http://www.webcitation.org/6wLMAkuaU); BioMed Central: ISRCTN10925965; https://www.isrctn.com/ISRCTN10925965 (Archived by WebCite at http://www.webcitation.org/6wLMVLuys)

(JMIR Res Protoc 2018;7(1):e8) doi: 10.2196/resprot.7720

\section{KEYWORDS}

randomized controlled trial; pelvic pain; chronic pain; mobile applications; mindfulness; meditation

\section{Introduction}

Female chronic pelvic pain (CPP) is defined as intermittent or constant pain in the lower abdomen or pelvis of a woman for at least 6 months, which is not exclusively associated with menstruation or intercourse, and not associated with pregnancy [1]. CPP affects up to $24 \%$ of women worldwide [2], accounts for $20 \%$ of gynecological clinic referrals in the United Kingdom [3], and has a considerable impact on patients' quality of life and their income. There are no recent estimates of the cost of CPP, but endometriosis-associated pain alone costs the United Kingdom economy $£ 8.2$ billion per year in treatment, loss of work, and health care costs [4], so the cost of general CPP is likely to be even higher. Despite costly interventions, CPP is often resistant to surgical and medical treatment. Both psychosocial causes (such as a history of sexual abuse) and somatic causes (eg, endometriosis, pelvic inflammatory disease, bladder pain syndrome) can contribute to CPP [1]. High levels of depression and anxiety are commonly associated with CPP, but are often not addressed in this population [5].

These multifactorial causes require a multidimensional approach, which is not routinely offered in gynecology clinics [6]. Evidence from randomized controlled trials (RCTs) suggests that a holistic approach using psychological interventions may be superior to primary surgery [7]. Although psychological treatment is provided across the National Health Service (NHS), mostly in the context of the primary care program Improving Access to Psychological Therapies, there are problems with capacity, waiting times, and the overall number of patients that are able to access services. Alternatively, supported self-management (SSM) is now recognized as a tool that empowers patients to better cope with their condition [8].

Mindfulness-based therapy is currently creating lively research interest. Two recent systematic reviews report positive effects on somatization disorders [9] and psychological stress [10]. A further systematic review carried out by our research team [11], which examined 15 RCTs for online mindfulness meditation, found small but significant beneficial impacts on depression, anxiety, well-being, and mindfulness, and a moderate effect on stress, with guided programs proving more effective than unguided ones. We are only aware of one ongoing randomized Danish study of mindfulness in patients with endometriosis-specific CPP (NCT02761382).

Our review also found that mindfulness meditation to treat CPP had a promising effect on patient well-being [11], as demonstrated in pilot studies on CPP and larger RCTs on other types of chronic pain. We are therefore investigating the feasibility of a full RCT for mindfulness in CPP, as mindfulness has great potential as a self-management tool that could be used as part of a holistic approach to CPP.

More convenient delivery methods have been called for as an alternative to the 8-week face-to-face sessions required for the standard mindfulness based stress reduction (MBSR) courses [12]. During a patient and public involvement (PPI) session we held to help design our study, CPP patients expressed a preference for receiving the intervention through a smartphone app, as it is portable and could be accessed when and where they liked.

The systematic review showed that mindfulness meditation helps chronic pain patients accept pain better and helps to alleviate anxiety and depression [11], which are particularly common in this population [5]. One of the suggested mechanisms of mindfulness meditation is the uncoupling of sensory aspects from the evaluative and emotional aspects of pain through mindful awareness and meditation. Unlike cognitive behavioral therapy, which is goal oriented, mindfulness meditation relies on nonjudgmental observation. By distancing themselves from painful sensations and thoughts, instead of being alarmed, patients can achieve greater acceptance of chronic pain rather than permanently wanting to control it.

\section{Systematic Review}

Our systematic search and review of the literature on mindfulness meditation in CPP (July 2013; updated May 2017) was designed to investigate prior research in the area before commencing our study. Our systematic review was conducted in line with current standards [13]. We searched MEDLINE (via OVID), EMBASE, PsychINFO, and AMED without language restrictions from database inception to July 2013, and subsequently updated the search in May 2017. The databases were searched for relevant studies using the following key words and word variants: chronic pain or pelvic pain, and meditation or mindfulness or Vipanassa or mindfulness based stress reduction or mindfulness based intervention or mindfulness based therapy. The reference lists from the obtained articles were examined for additional articles. We also hand-searched all relevant systematic reviews and, if necessary, we approached the authors for missing relevant information.

The first search identified two small, nonrandomized pilot trials investigating the effect of mindfulness meditation on pelvic pain $(n=22)$ [14], and endometriosis $(n=10)$ in particular [15]. Both small studies were uncontrolled. In the study on CPP, significantly improved scores were reported for daily maximum pain, physical function, mental health $(P=.01)$, and social function [14]. The mindfulness scores improved significantly in all measures [14]. In endometriosis patients, significant 
improvements were reported for bodily pain, general health, and vitality [15].

Since that time, two more studies have been published. Kanter et al investigated the effect of mindfulness compared to usual care in an RCT of patients with bladder pain syndrome $(n=20)$ [16]. Outcome measures relating to empowerment and self-management improved significantly [16]. A small pilot study on military women with CPP $(n=15)$ showed a nonsignificant reduction in pain and increase in mindfulness measures [12]. The authors of this study called for simpler formats of teaching mindfulness than the 8-week standard MBSR, which four studies used [12].

Given this paucity of data on mindfulness in CPP, we expanded our systematic review of mindfulness meditation to include its use in other chronic pain conditions (back pain, headaches, fibromyalgia, and diabetic neuropathy), as we assumed that any benefits in these conditions might also be seen in CPP. Previous systematic reviews of these conditions had a number of limitations, such as not reporting effect sizes [17-19].

We identified 534 relevant citations, and 9 RCTs [20-28] were included in the review [11]. Most studies were of moderate quality, but sample sizes were generally small (from 65 to 259 women). Our results showed mindfulness-based meditation reduced depression levels in chronic pain patients (standardized mean difference [SMD] $-0.31 ; 95 \%$ CI -0.52 to $-0.10 ; \mathrm{I}^{2}=0 \%$ ) and anxiety (SMD $-0.21 ; 95 \% \mathrm{CI}-0.45$ to $0.03 ; \mathrm{I}^{2}=0 \%$ ). Pain acceptance was also improved (SMD 0.34; 95\% CI 0.09-0.59). No significant changes were seen in quality of life, anxiety, pain scores, or the emotional response to pain.

There are few published robust trials of apps to assist better self-management of chronic conditions. A Cochrane review of apps for asthma found only two studies and concluded there was insufficient evidence to advise patients on their usefulness [29]. Although CPP is as common as back pain and asthma [30], there are no RCTs that are investigating mindfulness meditation in CPP. Mindfulness meditation had shown a promising effect on patient well-being in uncontrolled pilot studies on CPP and larger RCTs on other types of chronic pain [11]. Given the high levels of depression and anxiety in CPP patients, combined with difficult access to psychological treatments, this approach could address a gap both in knowledge and patient care.

\section{Development of the Mindfulness Meditation App Module}

Headspace [31], a company that had already developed and successfully established a mindfulness meditation app, was approached to develop a module for meditation for chronic pain. This module was incorporated into the existing library of app content. The pain module can be accessed after participants have completed the 10-day foundation program.

\section{Aims and Objectives}

The overall aim of this study is to assess the feasibility of implementing a full scale, multicenter RCT to test the efficacy of a mindfulness meditation intervention delivered by a mobile phone app for patients with CPP. The primary objectives are: (1) to provide feasibility data for a large multicenter RCT aimed at rigorously testing mindfulness meditation in patients with CPP (the full-scale trial will assess the effectiveness of the mindfulness meditation app in patients with CPP in a national multicenter RCT); and (2) to determine whether this app can be seamlessly integrated into clinical practice, especially CPP pathways.

\section{Methods}

\section{Design}

Mindfulness Meditation for Women With Chronic Pelvic Pain (MEMPHIS) is a three-arm randomized feasibility trial. Approval was received by Camden and Kings Cross Ethics Committee in February 2016 (15/LO/1967).

\section{Inclusion Criteria}

To be eligible for the MEMPHIS study, women were required to meet the following criteria: (1) aged 18 years or over; (2) have organic and nonorganic CPP lasting for 6 months or more; (3) be capable of understanding the information provided, and be able to understand simple English as is used in the app; and (4) give written informed consent.

\section{Exclusion Criteria}

Patients who met the following criteria were ineligible to participate: (1) no access to a personal computer or smartphone, or (2) current users of the Headspace app content available to the public.

\section{Study Design}

MEMPHIS is a three-arm randomized feasibility trial. All eligible women referred to the CPP clinics at the Royal London and Whipps Cross Hospitals (both new and existing patients) were approached to take part in the study. The setting of the study was NHS Tertiary care hospitals. After informed consent, we randomized eligible women in a 1:1:1 ratio (30 participants in each group) to one of the three treatment groups: (1) Intervention, consisting of 60 days of the app delivering mindfulness meditation content (in addition to usual care); (2) Active control, consisting of 60 days of the app delivering progressive muscle relaxation content (in addition to usual care); and (3) Treatment as usual consisting of usual care. See Figure 1 for a flow chart of the study.

\section{Outcomes}

\section{Feasibility Outcomes Collected From Participants}

Several parameters were collected regarding the participants (Textbox 1).

\section{Participant Focus Groups}

Usability and integration into clinical practice were explored in two postintervention focus groups at each recruiting site: one for each app group. All participants allocated to each app group were invited to a session to take place in a private room at or near the clinic they were recruited from. We aimed to recruit approximately 15 app participants from the study participants. We offered a telephone interview as an alternative to any participant who was unable to attend their focus group. 
Discussions were structured around the app System Usability Scale (SUS) and additional questionnaire, and were expected to take two hours to complete. Discussions were recorded and literal themes on integration and usability will be evaluated for in-depth information. This information will be considered, as well as adherence to the app, as an indirect measure of acceptability. We will determine primary and secondary outcomes of interest, from the perspective of patients, for a full-scale trial. This aim will involve asking participants who were randomized to the app groups to discuss and prioritize outcomes. Obstacles to recruitment will also be explored.

\section{Health Care Practitioner Focus Groups}

The health care practitioners that were involved with the trial were invited to attend focus groups. A purpose-made topic guide was used to structure focus groups with service providers, which were held in private rooms at the university or clinic, and based on the Normalization Process Theory toolkit [34] and Diffusion of Innovations Theory [35]. Topics were used as a prompt by the facilitator to identify any emergent or residual problems that might act as a barrier to use and effectiveness of the app in the RCT, and implementation into practice.
The service providers were asked to consider their role and their organization, to suggest and discuss any issues related to integration, and to also suggest potential solutions (unlike conventional qualitative research focus groups). Discussions considered relative advantage versus existing practices, compatibility with existing practices, simplicity and ease of integration, trialability and reinvention of the process, feedback (eg, can clinicians see that patients benefit?), and peer-to-peer networking. We will use our findings to develop our integration approach to be further explored in the subsequent full trial. Obstacles to recruitment will also be explored.

\section{Clinical Outcomes}

Clinical outcomes measures have been selected based on the findings of our systematic review [11] and for their relevance to women with CPP. All scales are validated. We will use the clinical outcomes listed in Textbox 2.

All clinical outcomes were self-reports completed by study participants at baseline, 60 days, 3 months, and 6 months postrandomization (Multimedia Appendix 2).

Figure 1. Participant flow showing eligibility, enrolment, randomization and follow-up.

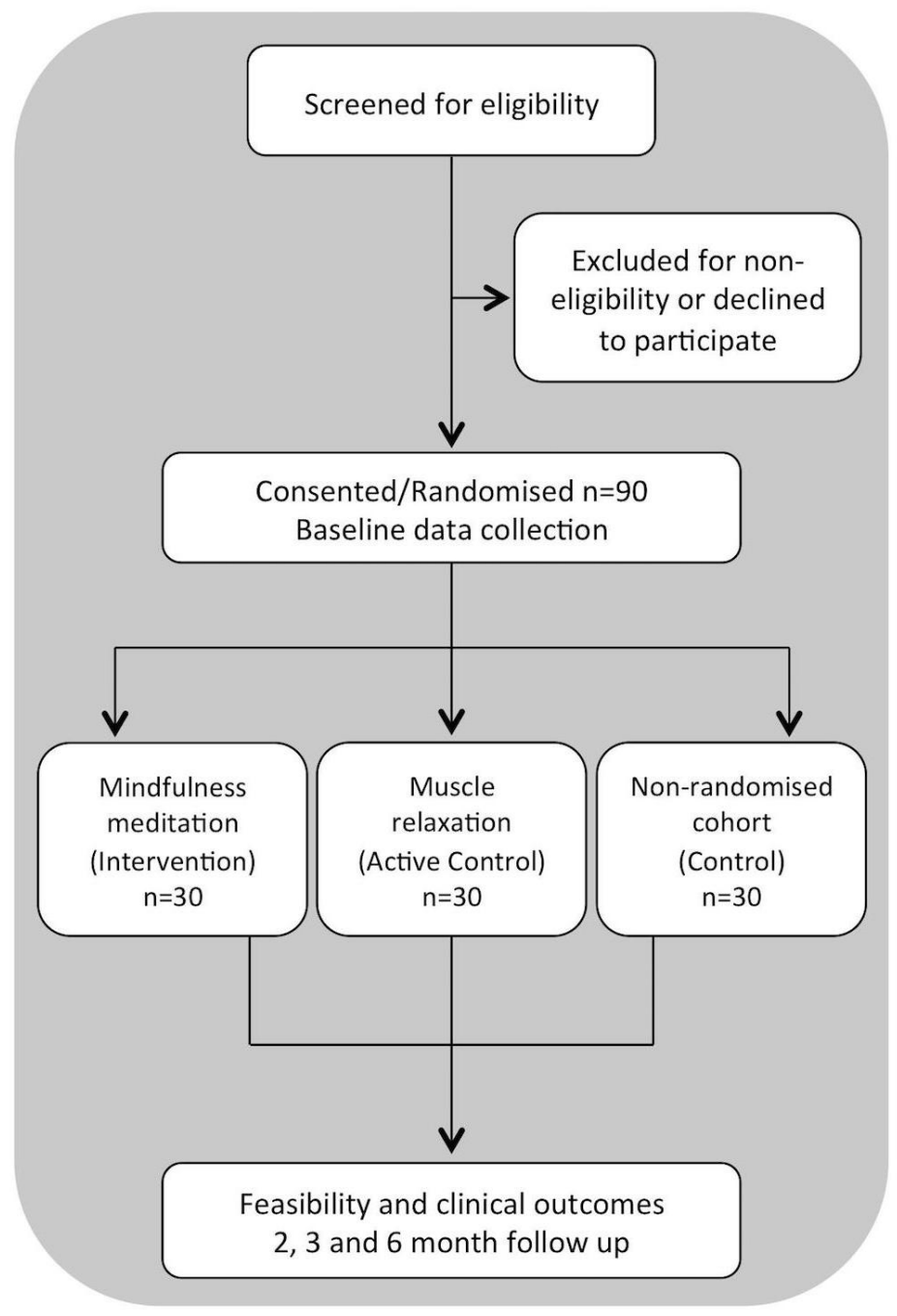


Textbox 1. Feasibility outcomes collected from patients

- $\quad$ Time taken to recruit all patients for the study (measured from the day recruitment opens until the day the 90th patient was randomized).

- Estimates to be used for the sample size calculation of the full-scale randmoized control trial:

- The estimated standard deviation for pain acceptance (as measured by the Chronic Pain Acceptance Questionnaire [CPAQ-8] [32])

- The dropout rate defined by the proportion of participants who never return or answer a follow-up questionnaire at 6 months postrandomization

- The proportion of participants who do not return a follow-up questionnaire, but do answer the questionnaire by phone at 6 months postrandomization

- $\quad$ Patient adherence to app use will be investigated using the following outcomes:

- Number of days (within the first 60 days from randomization) a patient has used the app. The definition of app use was originally defined as having completed $50 \%$ of a session. This definition was changed to $90 \%$ of a session during the trial (see Multimedia Appendix 1).

- Whether the patient has used the app on 22 or more days within the first 60 days following randomization

- Number of weeks (within the first 8 weeks from randomization) during which a patient has used the app on three or more days

- Whether the patient has used the app on three or more days in 6 or more weeks (within the first 8 weeks from randomization)

- Whether the patient has used the app on 22 or more days within the first 60 days from randomization, and used the app on three or more days in 6 or more weeks within the first 8 weeks from randomization

- Usability of the app will be measured by:

- $\quad$ The System Usability Scale [33]

- A purpose-made nonvalidated questionnaire (Multimedia Appendix 2) developed from patient and public involvement discussion and exploring the issues this group suggested women with chronic pelvic pain might have when using the particular app chosen. For example, we consider its modular format as well as the fit within daily activities, as we had been told from public and patient engagement work that this might be a problem for working women.

Textbox 2. Clinical outcomes

- $\quad$ Pain acceptance score (as measured by the Chronic Pain Acceptance Questionnaire [CPAQ-8] [32])

- Quality of life score, Physical Functioning subscale (as measured by the RAND 36-item Short Form health survey [SF-36] [36])

- Quality of life score, Social Functioning subscale (as measured by the RAND SF-36)

- Quality of life score, Pain subscale (as measured by the RAND SF-36)

- Quality of life score, General Health subscale (as measured by the RAND SF-36)

- Depression score (as measured by the Hospital Anxiety and Depression Scale [HADS] [37])

- Anxiety score (as measured by the HADS)

- $\quad$ Mindfulness score (as measured by the Cognitive and Mindfulness Scale - Revised [CAMS-R] [38])

- $\quad$ Pain related disability score (as measured by the Chronic Pain Grade [CPG] disability subscale [39])

- $\quad$ Self-efficacy score (as measured by the Pain Self-Efficacy Questionnaire [PSEQ] [40])

- Sexual Health Outcomes score (as measured by Sexual Health Outcomes in Women Questionnaire [SHOW-Q] [41])

- $\quad$ Subjective outcome score (as measured by Measure Yourself Medical Outcome Profile [MYMOP] [42])

\section{Recruitment}

\section{Informed Consent Procedures}

Women were made aware of the study by health care professionals and through promotional materials. Potentially eligible patients received the Patient Information Sheet (PIS) and were given adequate time (at least 24 hours) to consider the trial. Health care professionals and researchers screened eligibility at the time of outpatient clinic visits before offering participation. Women not interested or not eligible were recorded in a screening log. Eligible patients who were seen in clinics other than pelvic pain and endometriosis clinics were given the PIS and contact details for the researcher so they could benefit from participating in MEMPHIS, if they so wished.

\section{Randomization Procedures}

After providing informed consent, patients were randomized (maintaining full allocation concealment) in a 1:1:1 ratio to one of the three treatment groups, using permuted blocks without stratification. Block sizes were 27, 30, and 33. Randomization was performed using a centralized Internet service, hosted by the Pragmatic Clinical Trials Unit at Queen Mary University of London. 


\section{Blinding Following Allocation to Study Arm}

The participant and recruiting staff were aware of allocation to either the Treatment as usual group or one of the app groups. The participant and recruiting staff were, however, blinded to allocation to the Intervention or Active Control app groups.

To preserve blinding of participants within the two app arms of the study, each group used the same app and hearing instructions for the same duration, delivered by the same narrator. Only the content of the instructions differed. In addition, the PIS and consent forms did not explicitly refer to "mindfulness meditation" or "progressive muscle relaxation."

Data on whether any unblinding occured with recruiting staff, or if participants were aware of their allocation, were collected immediately after randomization and after 6 months, respectively. Statisticians will be blinded to individual treatment allocations until required for the final analysis.

\section{Interventions}

The researchers encouraged participants to aim to use the app they were randomized to daily, for as many sessions as they felt comfortable with.

\section{The Active Intervention: Mindfulness Meditation App}

The meditation content was delivered via a structured and progressive course, layering in new techniques and concepts over successive sessions. The course was created and narrated by a former monk, Andy Puddicombe, drawing on a secularized version of the techniques he was taught over 10 years in monasteries around the world.

The techniques used in the Intervention are shown in Table 1. The first 30 days covered basic techniques, assuming no previous experience of meditation. The second 30 days focused specifically on the use of these techniques with respect to pain. The duration of individual sessions built over time. Days 1-10 involved 10 minutes/day, days 11-20 involved 15 minutes/day, and days 21-60 involved 20 minutes/day. Headspace collected data on adherence to the active intervention.

\section{The Active Control App and the Treatment As Usual Group}

The Active Control group used the same app, but the app was configured so that they heard a series of nonmeditative progressive muscle relaxation instructions, also narrated by Andy Puddicombe. These sessions were identical every day, except that their duration increased to mirror the increasing duration of the meditation content being listened to by the Intervention group.
In this way, both Intervention and Active Control groups used the same app, and hearing instructions for the same duration, delivered by the same narrator. Only the content of the instructions differed. Headspace collected data on adherence to the active control.

The control group consisting of treatment as usual (watch and wait, medication, and/or surgery) was included to investigate if any app intervention makes a difference to well-being and to ascertain dropout rates for the full-scale trial in patients who perceive that they are receiving no intervention.

\section{Schedule of Assessment}

Health outcome measures were collected at baseline and immediately after the intervention at 60 days, 3 months, and 6 months. The time points were chosen to obtain information close to finishing the intervention to assess immediate effects, at a mid-point, and after 6 months to assess longer-term effects. App satisfaction/usability measures were collected immediately after the intervention (at 60 days) from app participants. Patients entered the data on paper questionnaires, which was transferred into a purpose-built electronic database. Data management will be carried out using OpenClinica Enterprise v3.12.2.

As an incentive to complete and return the patient questionnaires, a $£ 5$ shopping voucher was sent in the post with each follow-up questionnaire, alongside a stamped addressed envelope. In the case that a questionnaire was not received, participants were sent a text message reminder. Nonresponders were then contacted by telephone in order to collect a subset of the questionnaire.

Participants allocated to the app groups were asked for permission to elaborate on the open comment boxes about app satisfaction, and discuss and prioritize clinical outcomes in two focus groups. The focus groups were held after the 6-month follow-up point finished. If participants were unable to attend a focus group, they were given the option to take part in a phone interview.

\section{Statistical Analyses}

A full analysis plan can be found in Multimedia Appendix 3. Thirty participants were recruited to each of the three treatment groups, giving a total of 90 participants. As this is a feasibility study, we have not performed a sample size calculation based upon the power to detect a significant treatment effect on a clinical outcome. However, 90 participants should provide a reliable estimate for the standard deviation (SD) of the primary clinical outcome (likely to be pain acceptance) [43,44], which can be used to inform the sample size calculation of the main trial. Statistical analyses will be carried out using Stata 14.

Table 1. Meditation content over 60-day progressive course.

\begin{tabular}{ll}
\hline Series & Techniques involved \\
\hline Take 10/Foundation 1 (first 10 days) & Open monitoring, body scan, breath as anchor \\
Foundation 2 (days 11-20) & As above, plus intention and altruism \\
Foundation 3 (days 21-30) & As above, plus integration of mindfulness with daily activities \\
Pain series (days, 31-60) & As above, plus visualization and enquiry (insight/Tibetan Vipassana) \\
\hline
\end{tabular}




\section{Baseline}

Baseline variables will be summarized for each treatment group using descriptive statistics.

\section{Analysis of Feasibility Outcomes}

The time taken to recruit all patients for the study and the number of participants recruited per month will be presented. An estimate of the SD of pain acceptance (CPAQ-8) in each treatment group at each follow up time point (60 days, 3 months, and 6 months) will be presented.

The proportion of patients in each treatment group who returned data at each follow-up time point (60 days, 3 months, and 6 months postrandomization) will be summarized. Summaries of baseline variables will be presented separately for patients who did and did not return data at each at the 6-month time point. Patient adherence outcomes and outcomes measuring usability of the app will be summarized separately for the intervention and active control treatment groups using descriptive statistics.

\section{Analysis of Clinical Outcomes}

For each clinical outcome we will present the number of patients in each treatment group with an observed outcome at each follow-up time point, and the mean (SD) in each treatment group at each follow-up time point. Estimates of treatment effect and 95\% confidence intervals will be presented comparing the intervention group (mindfulness meditation app) to the control (treatment as usual) group, the intervention group to the active control (progressive muscle relaxation app) group, and the active control group to the control (treatment as usual) group.

Outcomes will be analyzed using linear mixed-effects models with outcome measurement (at three follow-up time points) as the dependent variable. The model will include fixed time effects, a fixed effect for treatment, time treatment interactions for 3-month and 6-month follow-up time points, and an unstructured correlation matrix for the residuals [45]. The model will include a baseline measure of the outcome as a covariate, assuming a linear relationship between baseline and outcome [46]. The model will be fitted using restricted maximum likelihood. Patient data will be analyzed according to the treatment group to which they were randomized (intention-to-treat). All patients with an observed outcome for at least one of the three follow-up time points (60 days, 3 months, or 6 months) will be included in the analysis [47]. If there are missing values for baseline measures of a clinical outcome, they will be replaced by the mean of the observed baseline values for all participants in all treatment arms (mean imputation) [48].

\section{Qualitative Analysis}

We will undertake a literal thematic analysis of the data from the focus group discussions to help us understand usability and implementation of, and response to, the intervention and research protocols $[49,50]$ rather than developing or testing theory [51]. Features of app use and implementation issues will be summarized in a table of app features. For example, we will populate the row labelled modular design with comments on this specific feature. Columns of this table will represent more granular themes.

\section{Results}

Recruitment for MEMPHIS took place between May 2016 and September 2016. The study was closed March 2017 and the report was submitted to the NIHR on October 26, 2017.

\section{Discussion}

There are currently no rigorous RCTs that test mindfulness meditation interventions as a therapy for any chronic pain syndrome, including CPP. A previous systematic review [52] and our systematic review [11] identified no RCTs on mindfulness meditation in CPP. However, recent pilot studies $[12,14,15]$ demonstrate promising outcomes and open the door for a large well-designed study with meaningful outcome measures. Given that psychological approaches, when combined with traditional therapies such as surgery, improve outcomes in CPP [7], mindfulness meditation is worthy of further investigation.

The intervention under investigation is novel in that it makes use of mindfulness meditation techniques as a treatment for CPP and it delivers this by means of a smartphone app (rather than traditional face-to-face therapy). Web- and smartphone-based health apps are a burgeoning field and offer the nonmedical population assistance in self-diagnosis, monitoring of long term medical conditions, or learning healthy behaviors. In the pain field alone, 279 smartphone apps were available for download in 2014, but these were simplistic, had unverified efficacy, and lacked the involvement of health care professionals in their development [53]. In our experience, no apps are currently incorporated into widespread routine clinical practice in CPP management. MEMPHIS presents a valuable opportunity to create a partnership between app development and health care professionals.

Not all patients with CPP would be expected to be highly confident in using smartphones and mobile technology, especially since women who are not usually using apps were recruited. Part of our data analysis, collected through focus groups, is directed towards the acceptability and usability of the app and will be valuable for any researcher planning future trials of smartphone technology in clinical interventions.

Given the ubiquity of the app, greater compliance with treatment and less wastage from patients not attending appointments may be expected. The use of the app in local primary, secondary, and tertiary care settings would be introduced in collaboration with general practitioner commissioning groups through local guidelines and protocols. Finally, if the app is shown to be effective in a full-scale trial, there would be benefit from studying how to extend the app to other pain conditions, such as headaches, back pain, and irritable bowel syndrome, in which face-to-face delivery of mindfulness meditation has had positive effects [9]. 


\section{Acknowledgments}

This protocol presents independent research funded by the National Institute for Health Research (NIHR) under its Research for Patient Benefit Program (Grant Reference Number PB-PG-1013-32025). SJCT was supported by the NIHR Collaboration for Leadership in Applied Health Research and Care North Thames at Bart's Health NHS Trust. The views expressed are those of the author(s) and not necessarily those of the NHS, the NIHR, or the Department of Health.

\section{Conflicts of Interest}

None declared.

\section{Multimedia Appendix 1}

Amendments to protocol.

[PDF File (Adobe PDF File), 19KB-Multimedia Appendix 1]

\section{Multimedia Appendix 2}

Case report forms to be used for collection of clinical outcome data.

[PDF File (Adobe PDF File), 434KB-Multimedia Appendix 2]

\section{Multimedia Appendix 3}

Statistical analysis plan.

[PDF File (Adobe PDF File), 347KB-Multimedia Appendix 3]

\section{Multimedia Appendix 4}

National Institute for Health Research peer review document.

[PDF File (Adobe PDF File), 131KB-Multimedia Appendix 4]

\section{References}

1. Moore SJ, Kennedy SH. The Royal College of Obstetricians and Gynaecologists. 2012 May. Green top guideline No 41: the initial management of chronic pelvic pain URL: https://www.rcog.org.uk/globalassets/documents/guidelines/gtg 41. pdf [accessed 2017-12-20] [WebCite Cache ID 6p0c0wzJx]

2. Latthe P, Latthe M, Say L, Gülmezoglu M, Khan KS. WHO systematic review of prevalence of chronic pelvic pain: a neglected reproductive health morbidity. BMC Public Health 2006 Jul 06;6:177 [FREE Full text] [doi:

10.1186/1471-2458-6-177] [Medline: 16824213 ]

3. Howard FM. The role of laparoscopy in chronic pelvic pain: promise and pitfalls. Obstet Gynecol Surv 1993 Jun;48(6):357-387. [Medline: $\underline{8327235}$ ]

4. Simoens S, Dunselman G, Dirksen C, Hummelshoj L, Bokor A, Brandes I, et al. The burden of endometriosis: costs and quality of life of women with endometriosis and treated in referral centres. Hum Reprod 2012 May;27(5):1292-1299. [doi: 10.1093/humrep/des073] [Medline: 22422778]

5. Romão AP, Gorayeb R, Romão GS, Poli-Neto OB, dos Reis FJ, Rosa-e-Silva JC, et al. High levels of anxiety and depression have a negative effect on quality of life of women with chronic pelvic pain. Int J Clin Pract 2009 May;63(5):707-711. [doi: 10.1111/j.1742-1241.2009.02034.x] [Medline: 19392920]

6. Stones RW, Price C. Health services for women with chronic pelvic pain. J R Soc Med 2002 Nov;95(11):531-535 [FREE Full text] [Medline: 12411615]

7. Peters AA, van Dorst E, Jellis B, van Zuuren E, Hermans J, Trimbos JB. A randomized clinical trial to compare two different approaches in women with chronic pelvic pain. Obstet Gynecol 1991 May;77(5):740-744. [Medline: 1826544]

8. Warsi A, Wang PS, LaValley MP, Avorn J, Solomon DH. Self-management education programs in chronic disease: a systematic review and methodological critique of the literature. Arch Intern Med 2004;164(15):1641-1649. [doi: 10.1001/archinte.164.15.1641] [Medline: 15302634]

9. Lakhan SE, Schofield KL. Mindfulness-based therapies in the treatment of somatization disorders: a systematic review and meta-analysis. PLoS One 2013;8(8):e71834 [FREE Full text] [doi: 10.1371/journal.pone.0071834] [Medline: 23990997]

10. Goyal M, Singh S, Sibinga EMS, Gould NF, Rowland-Seymour A, Sharma R, et al. Meditation programs for psychological stress and well-being: a systematic review and meta-analysis. JAMA Intern Med 2014 Mar;174(3):357-368 [FREE Full text] [doi: 10.1001/jamainternmed.2013.13018] [Medline: 24395196] 
11. Ball EF, Nur SMSE, Franklin G, Rogozińska E. Does mindfulness meditation improve chronic pain? A systematic review. Curr Opin Obstet Gynecol 2017 Dec;29(6):359-366. [doi: 10.1097/GCO.0000000000000417] [Medline: 28961631]

12. Crisp CD, Hastings-Tolsma M, Jonscher KR. Mindfulness-based stress reduction for military women with chronic pelvic pain: a feasibility study. Mil Med 2016 Sep;181(9):982-989. [doi: 10.7205/MILMED-D-15-00354] [Medline: 27612341]

13. Higgins J, Green S. Cochrane Handbook for Systematic Reviews of Interventions Version 5.1.0. 2011 Mar. URL: http:/ /handbook.cochrane.org [accessed 2017-12-20] [WebCite Cache ID 6p0fuCjRl]

14. Fox SD, Flynn E, Allen RH. Mindfulness meditation for women with chronic pelvic pain: a pilot study. J Reprod Med 2011;56(3-4):158-162. [Medline: 21542535]

15. Kold M, Hansen T, Vedsted-Hansen H, Forman A. Mindfulness-based psychological intervention for coping with pain in endometriosis. Nord Psychol 2012 Aug 31;64(1):2-16. [doi: 10.1080/19012276.2012.693727]

16. Kanter G, Komesu YM, Qaedan F, Jeppson PC, Dunivan GC, Cichowski SB, et al. Mindfulness-based stress reduction as a novel treatment for interstitial cystitis/bladder pain syndrome: a randomized controlled trial. Int Urogynecol J 2016 Nov;27(11):1705-1711. [doi: 10.1007/s00192-016-3022-8] [Medline: 27116196]

17. Cramer H, Haller H, Lauche R, Dobos G. Mindfulness-based stress reduction for low back pain. A systematic review. BMC Complement Altern Med 2012;12:162 [FREE Full text] [doi: 10.1186/1472-6882-12-162] [Medline: 23009599]

18. Kozasa EH, Tanaka LH, Monson C, Little S, Leao FC, Peres MP. The effects of meditation-based interventions on the treatment of fibromyalgia. Curr Pain Headache Rep 2012 Oct;16(5):383-387. [doi: 10.1007/s11916-012-0285-8] [Medline: 22717699]

19. Veehof MM, Oskam M, Schreurs KM, Bohlmeijer ET. Acceptance-based interventions for the treatment of chronic pain: a systematic review and meta-analysis. Pain 2011 Mar;152(3):533-542 [FREE Full text] [doi: 10.1016/j.pain.2010.11.002] [Medline: 21251756]

20. Brown CA, Jones AK. Psychobiological correlates of improved mental health in patients with musculoskeletal pain after a mindfulness-based pain management program. Clin J Pain 2013 Mar;29(3):233-244. [doi: 10.1097/AJP.0b013e31824c5d9f] [Medline: 22874090]

21. Carson JW, Keefe FJ, Lynch TR, Carson KM, Goli V, Fras AM, et al. Loving-kindness meditation for chronic low back pain: results from a pilot trial. J Holist Nurs 2005 Sep;23(3):287-304. [doi: 10.1177/0898010105277651] [Medline: $\underline{16049118]}$

22. Morone NE, Greco CM, Weiner DK. Mindfulness meditation for the treatment of chronic low back pain in older adults: a randomized controlled pilot study. Pain 2008 Feb;134(3):310-319 [FREE Full text] [doi: 10.1016/j.pain.2007.04.038] [Medline: 17544212]

23. Nash-McFeron D. Mindfulness in the treatment of chronic headache pain. Dissertation Abstracts International Section B 2006;67(5-B):2841.

24. Plews-Ogan M, Owens JE, Goodman M, Wolfe P, Schorling J. A pilot study evaluating mindfulness-based stress reduction and massage for the management of chronic pain. J Gen Intern Med 2005 Dec;20(12):1136-1138 [FREE Full text] [doi: 10.1111/j.1525-1497.2005.0247.x] [Medline: 16423104]

25. Sagula D, Rice KG. The effectiveness of mindfulness training on the grieving process and emotional well-being of chronic pain patients. J Clin Psychol Med Settings 2004 Dec;11(4):333-342. [doi: 10.1023/B:JOCS.0000045353.78755.51]

26. Schmidt S, Naranjo J, Gmeiner S, Brenneisen C, Gundlach J, Schultz C, et al. Assessing a dynamical EEG pattern related to chronic pain: results from a controlled evaluation of back pain patients as well as from an observational trial with a mindfulness based intervention. Eur J Integr Med 2010 Dec;2(4):196. [doi: 10.1016/j.eujim.2010.09.051]

27. Sephton SE, Salmon P, Weissbecker I, Ulmer C, Floyd A, Hoover K, et al. Mindfulness meditation alleviates depressive symptoms in women with fibromyalgia: results of a randomized clinical trial. Arthritis Rheum 2007 Feb 15;57(1):77-85 [FREE Full text] [doi: 10.1002/art.22478] [Medline: 17266067]

28. Teixeira E. The effect of mindfulness meditation on painful diabetic peripheral neuropathy in adults older than 50 years. Holist Nurs Pract 2010;24(5):277-283. [doi: 10.1097/HNP.0b013e3181f1add2] [Medline: 20706089]

29. Marcano BJ, Huckvale K, Greenfield G, Car J, Gunn LH. Smartphone and tablet self management apps for asthma. Cochrane Database Syst Rev 2013;11:CD010013. [doi: 10.1002/14651858.CD010013.pub2] [Medline: 24282112]

30. Daniels JP, Khan KS. Chronic pelvic pain in women. BMJ 2010 Oct 05;341:c4834. [Medline: 20923840]

31. Headspace. 2018. URL: https://www.headspace.com/buy/

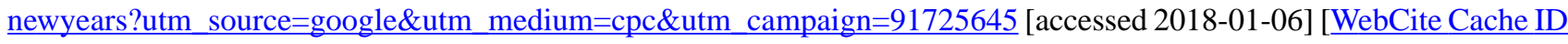
6wHAGNedM]

32. Fish RA, McGuire B, Hogan M, Morrison TG, Stewart I. Validation of the chronic pain acceptance questionnaire (CPAQ) in an Internet sample and development and preliminary validation of the CPAQ-8. Pain 2010 Jun;149(3):435-443. [doi: 10.1016/j.pain.2009.12.016] [Medline: 20188472]

33. Brooke J. SUS: a quick and dirty usability scale. In: Jordan PW, Thomas B, Weerdmeester BA, McCelland IL, editors. Usability Evaluation in Industry. London: Taylor \& Francis; 1996:189-194.

34. May C, Sibley A, Hunt K. The nursing work of hospital-based clinical practice guideline implementation: an explanatory systematic review using Normalisation Process Theory. Int J Nurs Stud 2014 Feb;51(2):289-299 [FREE Full text] [doi: $\underline{\text { 10.1016/j.ijnurstu.2013.06.019] [Medline: 23910398] }}$ 
35. Rogers EM. Diffusion of Innovations, 5th edition. New York: Free Press; 2003:221.

36. RAND Health. RAND 36-Item Short Form Survey (SF-36) Scoring Instructions. 2017. URL: https://www.rand.org/health/ surveys tools/mos/36-item-short-form/scoring.html [accessed 2017-03-19] [WebCite Cache ID 6p5hvtdZd]

37. Zigmond AS, Snaith RP. The hospital anxiety and depression scale. Acta Psychiatr Scand 1983 Jun;67(6):361-370. [Medline: $\underline{6880820]}$

38. Feldman G, Hayes A, Kumar S, Greeson J, Laurenceau J. Mindfulness and emotion regulation: the development and initial validation of the Cognitive and Affective Mindfulness Scale-Revised (CAMS-R). J Psychopathol Behav Assess 2006 Nov 7;29(3):177-190. [doi: 10.1007/s10862-006-9035-8]

39. Von Korff M, Ormel J, Keefe FJ, Dworkin SF. Grading the severity of chronic pain. Pain 1992 Aug;50(2):133-149. [Medline: 1408309]

40. Nicholas MK. The pain self-efficacy questionnaire: taking pain into account. Eur J Pain 2007 Feb;11(2):153-163. [doi: 10.1016/j.ejpain.2005.12.008] [Medline: 16446108]

41. Learman LA, Huang AJ, Nakagawa S, Gregorich SE, Kuppermann M. Development and validation of a sexual functioning measure for use in diverse women's health outcome studies. Am J Obstet Gynecol 2008 Jun;198(6):710.e1-8; discussion 710.e8. [doi: 10.1016/j.ajog.2008.03.036] [Medline: 18538161]

42. Paterson C, Britten N. In pursuit of patient-centred outcomes: a qualitative evaluation of the 'Measure Yourself Medical Outcome Profile'. J Health Serv Res Policy 2000 Jan;5(1):27-36. [doi: 10.1177/135581960000500108] [Medline: 10787584]

43. Lancaster GA, Dodd S, Williamson PR. Design and analysis of pilot studies: recommendations for good practice. J Eval Clin Pract 2004 May;10(2):307-312. [doi: 10.1111/j.2002.384.doc.x] [Medline: 15189396]

44. Teare MD, Dimairo M, Shephard N, Hayman A, Whitehead A, Walters SJ. Sample size requirements to estimate key design parameters from external pilot randomised controlled trials: a simulation study. Trials 2014 Jul 03;15:264 [FREE Full text] [doi: 10.1186/1745-6215-15-264] [Medline: 24993581]

45. Rabe-Hesketh S, Skrondal A. Multilevel and Longitudinal Modeling Using Stata, 3rd Edition. College Station, Texas: Stata Press; 2012.

46. Kahan BC, Jairath V, Doré CJ, Morris TP. The risks and rewards of covariate adjustment in randomized trials: an assessment of 12 outcomes from 8 studies. Trials 2014 Apr 23;15:139 [FREE Full text] [doi: 10.1186/1745-6215-15-139] [Medline: 24755011]

47. White IR, Horton NJ, Carpenter J, Pocock SJ. Strategy for intention to treat analysis in randomised trials with missing outcome data. BMJ 2011 Feb 07;342:d40 [FREE Full text] [Medline: 21300711]

48. White IR, Thompson SG. Adjusting for partially missing baseline measurements in randomized trials. Stat Med 2005 Apr 15;24(7):993-1007. [doi: 10.1002/sim.1981] [Medline: 15570623]

49. O'Cathain A, Goode J, Drabble SJ, Thomas KJ, Rudolph A, Hewison J. Getting added value from using qualitative research with randomized controlled trials: a qualitative interview study. Trials 2014 Jun 09;15:215 [FREE Full text] [doi: 10.1186/1745-6215-15-215] [Medline: 24913438]

50. O'Cathain A, Hoddinott P, Lewin S, Thomas KJ, Young B, Adamson J, et al. Maximising the impact of qualitative research in feasibility studies for randomised controlled trials: guidance for researchers. Pilot Feasibility Stud 2015;1:32 [FREE Full text] [doi: 10.1186/s40814-015-0026-y] [Medline: 27965810]

51. Eakin JM. Educating critical qualitative health researchers in the land of the randomized controlled trial. Qual Inq 2015 Dec 03;22(2):107-118. [doi: 10.1177/1077800415617207]

52. Reiner K, Tibi L, Lipsitz JD. Do mindfulness-based interventions reduce pain intensity? A critical review of the literature. Pain Med 2013 Feb;14(2):230-242. [doi: 10.1111/pme.12006] [Medline: 23240921]

53. Lalloo C, Jibb LA, Rivera J, Agarwal A, Stinson JN. "There's a pain app for that": review of patient-targeted smartphone applications for pain management. Clin J Pain 2015 Jun;31(6):557-563. [doi: 10.1097/AJP.0000000000000171] [Medline: 25370138]

\section{Abbreviations}

CAMS-R: Cognitive and Mindfulness Scale - Revised

CPAQ-8: Chronic Pain Acceptance Questionnaire

CPP: chronic pelvic pain

HADS: Hospital Anxiety and Depression Scale

MBSR: mindfulness based stress reduction

MEMPHIS: Mindfulness Meditation for Women With Chronic Pelvic Pain

NHS: National Health Service

NIHR: National Institute for Health Research

PIS: Patient Information Sheet

PPI: patient and public involvement

RCT: randomized controlled trial

SD: standard deviation 
SF-36: RAND 36-item Short Form health survey

SMD: standardized mean difference

SSM: supported self-management

SUS: System Usability Scale

Edited by G Eysenbach; submitted 19.03.17; peer-reviewed by $M$ Ashford, H Devan; comments to author 25.05.17; revised version received 26.08.17; accepted 27.09.17; published 15.01.18

Please cite as:

Ball E, Newton S, Kahan BC, Forbes G, Wright N, Cantalapiedra Calvete C, Gibson HAL, Rogozinska E, Rivas C, Taylor SJC, Birch J, Dodds $J$

Smartphone App Using Mindfulness Meditation for Women With Chronic Pelvic Pain (MEMPHIS): Protocol for a Randomized Feasibility Trial

JMIR Res Protoc 2018;7(1):e8

URL: http://www.researchprotocols.org/2018/1/e8/

doi: $10.2196 /$ resprot. 7720

PMID: 29335232

(CElizabeth Ball, Sian Newton, Brennan C Kahan, Gordon Forbes, Neil Wright, Clara Cantalapiedra Calvete, Harry A L Gibson, Ewelina Rogozinska, Carol Rivas, Stephanie J C Taylor, Judy Birch, Julie Dodds. Originally published in JMIR Research Protocols (http://www.researchprotocols.org), 15.01.2018. This is an open-access article distributed under the terms of the Creative Commons Attribution License (https://creativecommons.org/licenses/by/4.0/), which permits unrestricted use, distribution, and reproduction in any medium, provided the original work, first published in JMIR Research Protocols, is properly cited. The complete bibliographic information, a link to the original publication on http://www.researchprotocols.org, as well as this copyright and license information must be included. 